\title{
Multiband Software Defined Radar for Soil Discontinuities Detection
}

\author{
S. Costanzo, ${ }^{1}$ F. Spadafora, ${ }^{1}$ O. H. Moreno, ${ }^{2}$ F. Scarcella, ${ }^{1}$ and G. Di Massa ${ }^{1}$ \\ ${ }^{1}$ Dipartimento di Ingegneria Informatica, Modellistica, Elettronica e Sistemistica, Università della Calabria, 87036 Rende (CS), Italy \\ ${ }^{2}$ Facultad de Informatica y Electronica, Escuela Superior Politecnica de Chimborazo, Riobamba, EC060155, Ecuador
}

Correspondence should be addressed to S. Costanzo; costanzo@deis.unical.it

Received 30 May 2013; Accepted 24 June 2013

Academic Editor: Alvaro Rocha

Copyright (C) 2013 S. Costanzo et al. This is an open access article distributed under the Creative Commons Attribution License, which permits unrestricted use, distribution, and reproduction in any medium, provided the original work is properly cited.

A multiband Software Defined Radar based on orthogonal frequency-division multiplexing technique is proposed in this work for an accurate soil discontinuities detection, taking into account also the dispersive behavior of media. A multilayer soil structure is assumed as a validation test to demonstrate the effectiveness of the proposed approach, by accurately retrieving the unknown thicknesses and permittivities of the soil layers.

\section{Introduction}

Electromagnetic sensors such as ground penetrating radar (GPR) are largely adopted for the detection of buried land mines, unexploded ordnance, and soil discontinuities. The performance of these sensors strongly depends on the dielectric properties of the soil, which in turn are related to specific parameters, such as texture, bulk density, and water content. The accurate knowledge of soil dielectric constant is of primary importance in this kind of radar application and is particularly difficult to achieve in the presence of dispersive media, where the dielectric properties vary with frequency. The use of a multiband radar technique can be strongly helpful in this case, thus an approach in terms of Software Defined Radar (SDR) system can be adopted to face the problem. As a matter of fact, the use of SDR technology leads to significant advantages in terms of lowcost, compactness, easy implementation of radar functions, and in particular signal processing techniques. The potentialities of SDR systems have been discussed by the authors in $[1,2]$ for the case of singleband applications. In this work, a multiband SDR technology is proposed to implement a GPR system, in order to perform an accurate soil discontinuities detection. For the considered multiband application, the adoption of SDR concept provides strong advantages with respect to classical radars, due to the easy software implementation of all required hardware modules, such as mixers, filters, modulators/demodulators, and the easier integration into a unique compact system of all necessary signal processing operations. In particular, in the present work, the adoption of an orthogonal frequencydivision multiplexing (OFDM) signal processing technique [3-7] is discussed to implement a multiband radar system able to retrieve the frequency variations of soil permittivity.

The benefit of multicarrier radar signaling [8] has been well established in various application fields, such as remote sensing of wheatear forecasting [9], detection of buried objects [10], and interpretation of urban scenes [11]. In these works, the strong advantages of OFDM in terms of robustness against multipath fading, easy synchronization and equalization, and a high flexibility in the system design are clearly outlined.

Alternative multiband signal methods also exist in literature. For example, ultrashort pulses (USP) are often used to accomplish a good radar range resolution. However, the main problem of these signals is related to the strong attenuation of the average transmitted power, which can hinder the radar's normal operation modes. The attenuation issue can be controlled by the adoption of chirp waveforms [12], but in this case a nonregular signal (typically frequency modulated) is applied, while the main advantage of OFDM radar systems is just related to the adoption of regular signals leading to a potential primary active radar system available at zero additional spectrum usage [13]. 
In synthesis, apart from the aforementioned features, the main advantages deriving from the adoption of an OFDM radar can be summarized as follows:

(i) digital waveforms generation with the possibility of pulse-to-pulse shape variation;

(ii) narrowband jamming/interference possibility mitigation by simply turning off specific subbands;

(iii) noise-like waveforms for increased low probability of intercept/detection;

(iv) high resolution and good robustness against multipath;

(v) high spectral efficiency;

(vi) ability to transmit large amounts of image data in real time;

(vii) flexible usage of subbands to be adopted and used for Doppler/location/voice communication.

In order to validate the proposed multiband OFDMbased SDR approach, a test of multilayer structure is considered in this work and a multiband OFDM algorithm is applied to accurately reconstruct the thickness and the permittivities of the soil layers [14-16]. As reference simulations, the empirical models described in $[14,15]$ are adopted to predict the permittivity behavior in the frequency range between $0.3 \mathrm{GHz}$ and $18 \mathrm{GHz}$.

\section{Empirical Models of Soil Electrical Properties}

The dielectric properties of a soil depend on a number of factors, such as bulk density, sand percentage, clay and silt particles, density of soil particles, volumetric water content, temperature, and frequency of interest [14-16].

In this section, we summarize the results obtained from the two empirical models $[14,15]$ largely adopted in the literature for an accurate soil simulation.

The first model is tuned for the frequency range between $0.3 \mathrm{GHz}$ and $1.3 \mathrm{GHz}$ [14], while the second one [15] is calibrated between $1.4 \mathrm{GHz}$ and $18 \mathrm{GHz}$. In these models, a dielectric constant as a function of frequency can be retrieved by setting the sand and clay fraction and the volumetric water content.

The models calculate the real and the imaginary parts of the dielectric constant $\varepsilon$ of a particular soil, on the base of the following inputs:

(i) $\theta$ : volumetric soil water content;

(ii) $f$ : operating frequency;

(iii) $S$ : fraction of sand particles;

(iv) $C$ : fraction of clay particles;

(v) $\rho_{s}$ : density of soil particles (typically $2.66 \mathrm{~g} / \mathrm{cm}^{3}$ );

(vi) $\rho_{b}$ : bulk density of the soil (typically $1.66 \mathrm{~g} / \mathrm{cm}^{3}$ );

(vii) $\varepsilon_{f w}=\varepsilon_{f w}^{\prime}+j \varepsilon_{f w}^{\prime \prime}$ : dielectric constant of free water.
The real and imaginary parts $\varepsilon^{\prime}, \varepsilon^{\prime \prime}$ of soil dielectric constant $\varepsilon$ are given by the following:

$$
\begin{gathered}
\varepsilon^{\prime}=\left[1+\frac{\rho_{B}}{\rho_{s}}\left(\varepsilon_{s}^{\alpha}-1\right)+\theta^{\beta \prime} \varepsilon_{f w}^{\prime \alpha}-\theta\right]^{1 / \alpha}, \\
\varepsilon^{\prime \prime}=\left[\theta^{\beta \prime \prime} \varepsilon_{f w}^{\prime \prime \alpha}\right]^{1 / \alpha},
\end{gathered}
$$

where

$$
\begin{gathered}
\alpha=0.65 \\
\beta^{\prime}=1.2748-0.519 S-0.152 C \\
\beta^{\prime \prime}=1.33797-0.603 S-0.166 C, \\
\varepsilon_{f w}^{\prime}=\varepsilon_{w \text { inf }}+\frac{\varepsilon_{w \infty}-\varepsilon_{w 0}}{1+\left(2 \pi f \tau_{w}\right)^{2}}, \\
\varepsilon_{f w}^{\prime \prime}=\frac{2 \pi f \tau_{w}\left(\varepsilon_{w 0}-\varepsilon_{w \infty}\right)}{1+\left(2 \pi f \tau_{w}\right)^{2}}+\frac{\sigma_{\mathrm{eff}}}{2 \pi \varepsilon_{0} f} \frac{\left(\rho_{s}-\rho_{B}\right)}{\rho_{s}^{\theta}} .
\end{gathered}
$$

In (3), $\varepsilon_{0}$ is the free-space permittivity, $\varepsilon_{w 0}$ gives the dielectric constant of the water equal to 20.1 at $20^{\circ} \mathrm{C}, \varepsilon_{w \infty}$ is the high frequency limit of $\varepsilon_{f w}^{\prime}$ equal to 4.9 , and $\tau_{w}$ is the relaxation time of the water equal to $9.23 \times 10^{-12} \mathrm{~s}$ at $20^{\circ} \mathrm{C}$. The dielectric constant of the soil particles $\varepsilon_{s}$ and the effective soil conductivity $\sigma_{\text {eff }}$ are given by the following empirical formulas:

$$
\begin{gathered}
\varepsilon_{s}=\left(1.01+0.44 \rho_{s}\right)^{2}-0.062, \\
\sigma_{\text {eff } 18 \mathrm{GHz}}^{1.3 \mathrm{GHz}}=-1.645+1.939 \rho_{B}-2.013 S+1.594 C, \\
\sigma_{\text {eff } 1.3 \mathrm{GHz}}^{0.3 \mathrm{GHz}}=0.0467+0.2204 \rho_{B}-0.4111 S+0.6614 \mathrm{C} .
\end{gathered}
$$

In Figure 1, the behavior of the real and imaginary parts for the soil dielectric constant $\varepsilon$ is reported versus frequency. Discontinuities in the graphs can be observed as due to the combinations of the two empirical models. In the following sections, these will be adopted to simulate a multilayer soil as validation test for the OFDM multiband algorithm.

\section{OFDM Signal Processing Method}

OFDM is a combination of modulation and multiplexing techniques, which allows the transmission of complex modulation symbols obtained through discrete phase modulation (e.g., BPSK). The block diagram of the OFDM principle is schematically illustrated in Figure 2.

The symbols use orthogonal subcarriers with duration $T$, which are frequency spaced by the interval:

$$
\Delta f=\frac{1}{T}
$$

in order to avoid intercarrier interference. Each complex modulation symbol is expressed by the following:

$$
\Psi_{k}(t)=S_{k}(t) e^{-j 2 \pi\left(f_{k}\right) t},
$$




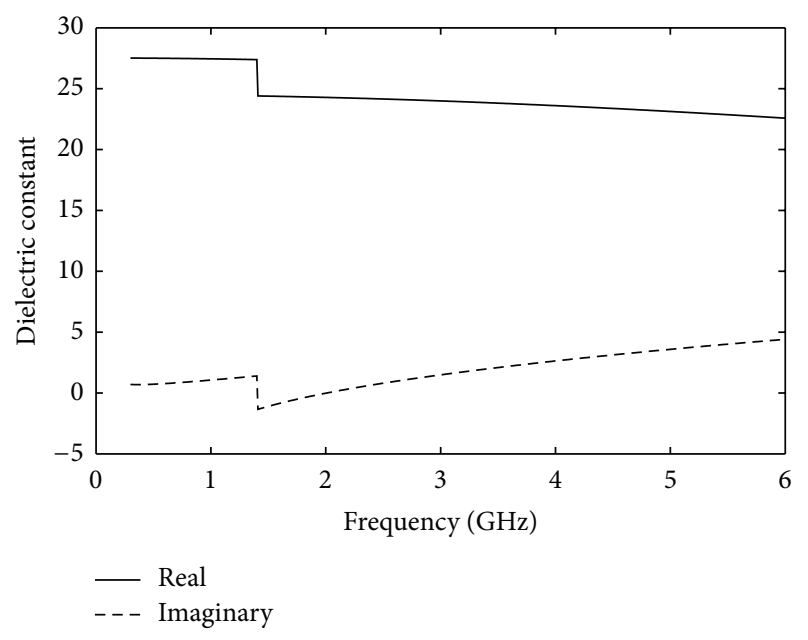

FIGURE 1: Dielectric constant versus frequency for a soil with $S=$ $0.95, C=0.02$, and $\theta=0.27$.

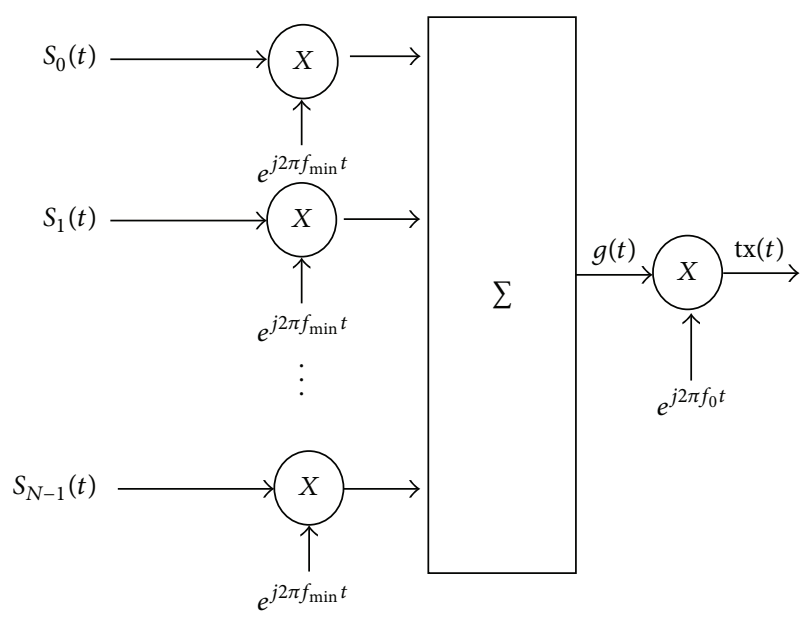

FIGURE 2: OFDM block diagram.

where $S_{k}(t)$ is a complex symbol (e.g., in BPSK it can be equal to 1 or -1 with duration $T$ ), and $f_{k}$ is the individual subcarrier frequency, given as

$$
f_{k}=f_{\min }+k \Delta f, \quad k=0,1,2, \ldots, N-1,
$$

with $f_{\min }$ giving the minimum frequency with an arbitrary value.

The multiplexed signal is represented as

$$
g(t)= \begin{cases}\sum_{k=0}^{N-1} S_{k}(t) e^{-j 2 \pi\left(f_{\min }+k \Delta f\right) t,} & 0<t<T \\ 0, & \text { otherwise. }\end{cases}
$$

It is modulated with a carrier frequency $f_{0}$ to obtain the OFDM signal, which is equal to

$$
\operatorname{tx}(t)=g(t) e^{j 2 \pi f_{0} t} .
$$

OFDM radar works as a static system, by transmitting a signal of kind (9) and receiving reflections of this signal from objects in the path of the signal's wavefront. It is important that transmitter and receiver are synchronized in order to avoid time or frequency offset. The received signal, in the absence of Doppler effect, can be expressed as

$$
\mathrm{rx}(t)=\sum_{h=0}^{H-1} b_{h} g\left(t-\tau_{h}\right) e^{j 2 \pi f_{0}\left(t-\tau_{h}\right)},
$$

where $H$ is the number of reflecting targets. Every target at a distance $d_{h}$ is represented by a delay $\tau_{h}$ of the corresponding signal. The term $b_{h}$ represents the attenuation for each target which depends on the material characteristics and the radar cross-section.

The OFDM signal processing technique gives a maximum measurement distance defined as

$$
d_{\max }=\frac{c}{2 \Delta f}=\frac{T c}{2}
$$

where $c$ is free-space velocity.

The radar slant range resolution depends on the total bandwidth $B$ occupied by the transmitted signal, so it is given by

$$
\Delta r=\frac{c}{2 B}=\frac{c}{2 N \Delta f}
$$

Figure 3 shows the block diagram for an OFDM radar, where the transmitted signal is composed by $N$ symbols $S=\left[S_{0}, S_{1}, S_{2}, \ldots, S_{N-1}\right]^{T}$ modulated with $N$ orthogonal frequencies $f=\left[f_{\min }, f_{\min }+\Delta f, f_{\min }+2 \Delta f, \ldots, f_{\min }+(N-\right.$ 1) $\Delta f]^{T}$ using OFDM technique. The total time $T_{t}$ for an OFDM symbol considers the symbol duration $T$ and a guard interval $T_{G}$, that is, the time to avoid intersymbol interference between subsequent OFDM symbols.

The received signal $\mathrm{rx}$ is demodulated, and a vector $Y=$ $\left[Y_{0}, Y_{1}, Y_{2}, \ldots Y_{1}\right]$, containing the values of received symbols, is retrieved.

The target position is then obtained by applying the IFFT on the result of the wise division between the symbols received $Y$ and that transmitted $S$.

\section{Multiband OFDM SDR}

The scheme reported in Figure 2 is relative to a single band OFDM system. In order to obtain a multiband configuration, the original block diagram is modified as in Figure 4, with the adoption of $M$ carrier frequencies, namely, $f_{01}, f_{02}, \ldots, f_{0 M}$.

There are two possibilities for obtaining an OFDM multiband signal tx. The first one consists in transmitting all the OFDM waves at the same time, using only one shoot of $T_{t}$ seconds, while in the second approach just one OFDM signal is transmitted each $T_{t}$ seconds, with a total time signal of $M * T_{t}$ seconds. The second option is the simplest one for processing and analysis, so it is adopted in this work for the reconstruction of multilayer soil permittivity. As an example, the spectrum of the a multiband OFDM signal with $M=5$ carriers is illustrated in Figure 5. 


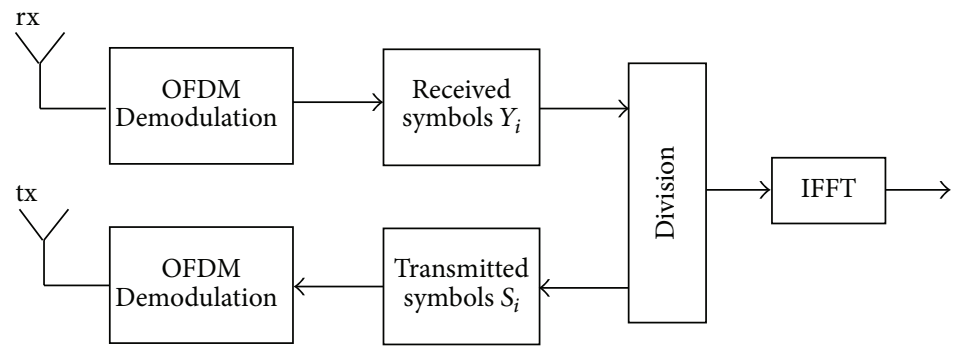

FIGURE 3: OFDM radar block diagram.

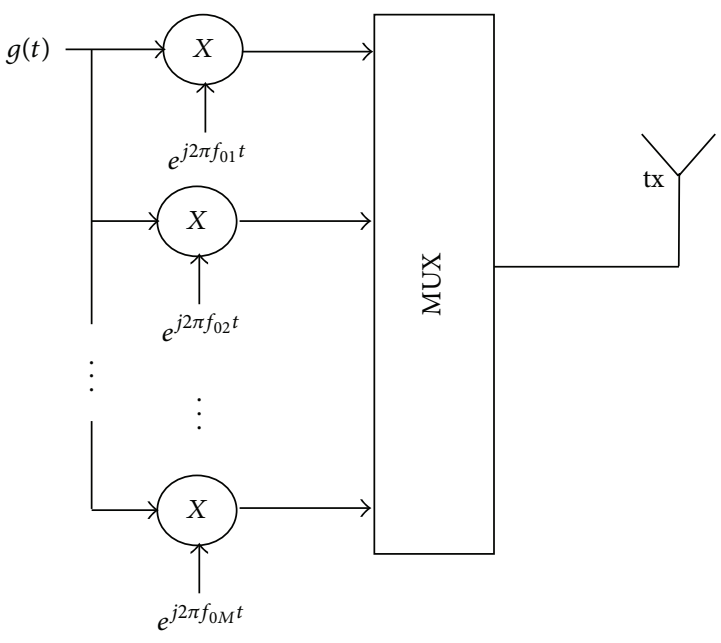

FIGURE 4: Block diagram of OFDM multiband radar system.

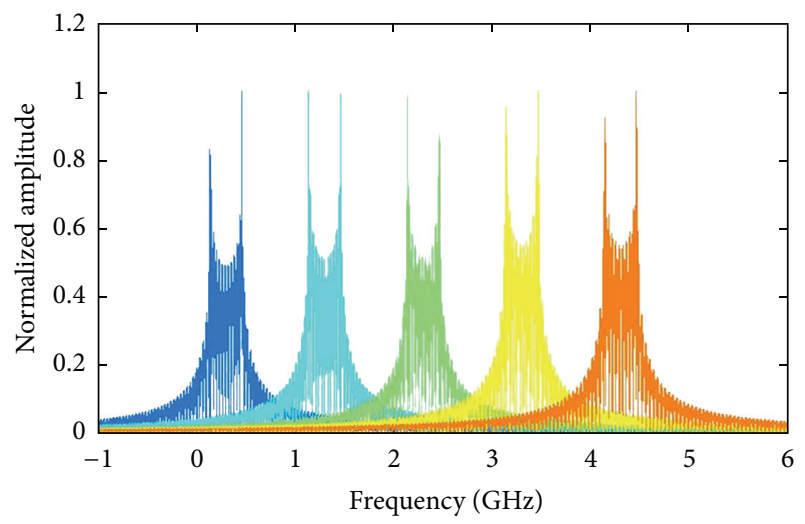

FIGURE 5: Spectrum of OFDM multiband signal with $M=5$ carriers.

\section{Dielectric Characterization Algorithm}

Let us consider a simple structure with 2 homogeneous materials having thickness $d_{1}$ and $d_{2}$, respectively (Figure 6). A radar signal from a distance $d_{0}>2 D^{2} / \lambda_{f_{\min }}\left(\lambda_{f_{\min }}\right.$ being the wavelength for the minimum frequency $f_{\min }$ under analysis) is assumed to impinge on the multilayer structure.

As a first case, we assume non-dispersive dielectric media, characterized by constant permittivities versus frequency. In this situation, we can use a single band OFDM signal tx $(t)$ to

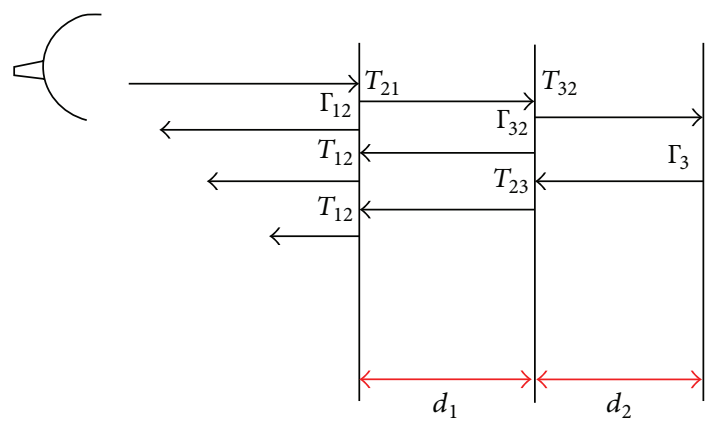

FIGURE 6: Multilayer test structure.

retrieve the permittivity values $\epsilon_{r 1}$ and $\epsilon_{r 2}$ of the two layers. The corresponding signal detected by the receiver is given by the following expression:

$$
\begin{aligned}
\mathrm{rx}(t)= & b_{0} g\left(t-\tau_{0}\right) e^{j 2 \pi f_{0}\left(t-\tau_{0}\right)} \\
& +b_{1} g\left(t-\tau_{1}\right) e^{j 2 \pi f_{0}\left(t-\tau_{1}\right)} \\
& +b_{2} g\left(t-\tau_{2}\right) e^{j 2 \pi f_{0}\left(t-\tau_{2}\right)},
\end{aligned}
$$

where

(i) $b_{0}$ is directly related to the reflection coefficient $\Gamma_{01}$ of the first reflection from the first substrate, and $\tau_{0}$ is the associated delay time;

(ii) $b_{1}$ is related to the expression $\left(1-\Gamma_{01}^{2}\right) \Gamma_{12}$ of the second reflection from the second substrate, and $\tau_{1}$ is the associated delay time;

(iii) $b_{2}$ is related to the expression $\left(1-\Gamma_{12}{ }^{2}\right) \Gamma_{23}$ of the third reflection from the third substrate, and $\tau_{2}$ is the associated delay time.

The terms $\Gamma_{01}, \Gamma_{12}$, and $\Gamma_{23}$ are well known in the literature [17].

The result of OFDM signal processing on the received signal (13) is illustrated in Figure 7, where the three peaks corresponding to the reflections from the three interfaces can be observed. In the same figure, the result obtained from a preliminary system calibration, performed with a perfect conductor surface covering the test structure, is also reported. 


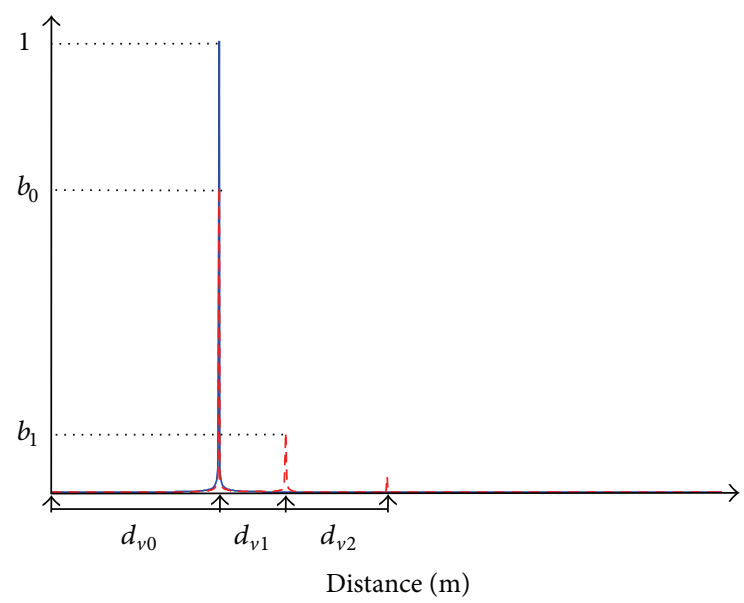

_ Radar range profile calibration

- - - Radar range profile of test structure

FIGURE 7: Radar range profile.

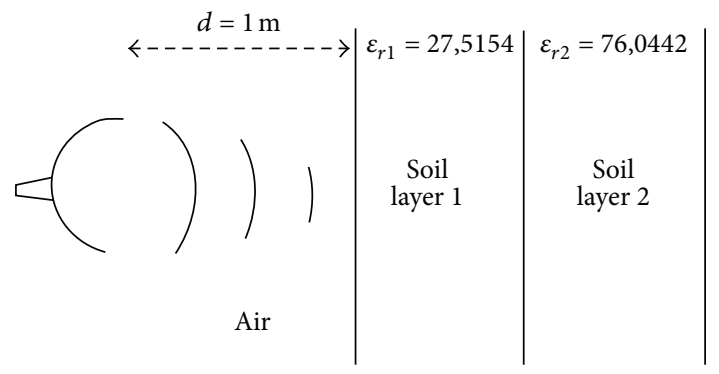

FIGURE 8: Simulated scenario.

A recursive algorithm is applied to retrieve the permittivity values $\epsilon_{r 1}$ and $\epsilon_{r 2}$. In particular, we have

$$
b_{0}=\Gamma_{01}=\frac{1-\sqrt{\epsilon_{r 1}}}{1+\sqrt{\epsilon_{r 1}}}
$$

from which it is straightforward to retrieve the value of $\epsilon_{r 1}$. Then, we can use the following expression:

$$
\frac{b_{1}}{b_{0}}=\frac{\left(1-\Gamma_{01}^{2}\right)}{\Gamma_{01}} \Gamma_{12}
$$

to retrieve the value of $\epsilon_{r 2}$ directly from $\Gamma_{12}$ and so on for the successive substrate:

$$
\frac{b_{2}}{b_{1}}=\frac{\left(1-\Gamma_{12}^{2}\right)}{\Gamma_{12}} \Gamma_{23} .
$$

A generalized equation for the algorithm can be expressed as

$$
\frac{b_{n}}{b_{n-1}}=\frac{\left(1-\Gamma_{n-1, n}^{2}\right)}{\Gamma_{n-1, n}} \Gamma_{n, n+1} .
$$

Once the values of the permittivity values $\epsilon_{r 1}$ and $\epsilon_{r 2}$ are known, the next step is to calculate the thicknesses $d_{1}$ and $d_{2}$ of the two layers. In particular, the distances $d_{v 0}, d_{v 1}$, and
TABLE 1: Input soil electrical model.

\begin{tabular}{lcc}
\hline & Soil layer 1 & Soil layer 2 \\
\hline$\theta$ & 0.27 & 0.8 \\
$S$ & 0.95 & 0.70 \\
$C$ & 0.02 & 0.01 \\
$\varepsilon_{r}$ & $\mathbf{2 7 . 5 1 5 4}$ & $\mathbf{7 6 . 0 4 4 2}$ \\
\hline
\end{tabular}

TABLE 2: Retrieved permittivities and thicknesses.

\begin{tabular}{lcc}
\hline & Soil layer 1 & Soil layer 2 \\
\hline Retrieved $\varepsilon_{r}$ & 26.8856 & 72.1654 \\
Retrieved layer thickness m & 0.3934 & 0.4453 \\
\hline
\end{tabular}

TABLE 3: Permittivity variation for each carrier frequency.

\begin{tabular}{lcc}
\hline Frequency $\mathrm{GHz}$ & Retrieved $\Delta \varepsilon_{r 1}$ & Retrieved $\Delta \varepsilon_{r 2}$ \\
\hline 2.3 & 1.0314 & 1.0503 \\
3.3 & 1.0314 & 1.0503 \\
4.3 & 1.0481 & 1.0726 \\
\hline
\end{tabular}

$d_{v 2}$ highlighted in Figure 7 are related to the real distances $d_{i}$ by the following relationship:

$$
d_{i}=\frac{d v i}{\sqrt{\epsilon_{r i}}}
$$

Let us assume, now, that layers in Figure 7 are composed by dispersive media, with a variation of permittivities versus frequency. In this case, a multiband OFDM technique can be adopted to retrieve the variation profile of dielectric constants. In particular, by considering $M$ different carriers as in Figure 4, the algorithm previously described for the case of single band OFDM must be repeated for every carrier frequency, thus obtaining $M$ graphs similar to that of Figure 7 but presenting a shift $\Delta d$, variable versus frequency, for the distances $d_{v 1}$ and $d_{v 2}$. It is in turn related to the permittivity shift $\Delta \varepsilon_{r}$, which can be expressed as

$$
\Delta \varepsilon_{r}=\left(\frac{d_{v i}}{d_{v i}-\Delta d}\right)^{2}
$$

\section{Numerical Results}

In order to validate the proposed multiband SDR technique, the multilayer soil structure in Figure 8 is assumed as a test scenario. The empirical models outlined in Section 2 are applied by assuming parameters $C$ (clay fraction), $S$ (sand fraction), and $\theta$ (water content) as reported in Table 1, where the simulated relative dielectric constants of the two layers are also indicated. As a first task, a single-band OFDM radar signal with $f_{0}=2.3 \mathrm{GHz}$ (Figure 2) is assumed to retrieve the values of $\epsilon_{r 1}, \epsilon_{r 2}$ and the thicknesses $d_{1}, d_{2}$ of the two layers (Table 2). In a successive step, a multiband OFDM signal with $M=3$ carriers is considered, thus obtaining the curves reported in Figure 9, for a frequency variation from $2.3 \mathrm{GHz}$ up to $4.3 \mathrm{GHz}$. The relative detected values of $\Delta d$, variable with carrier frequency (Figure 9), are then used to obtain the variation profile of the permittivity as a function of frequency, for the two soil layers (Table 3). 


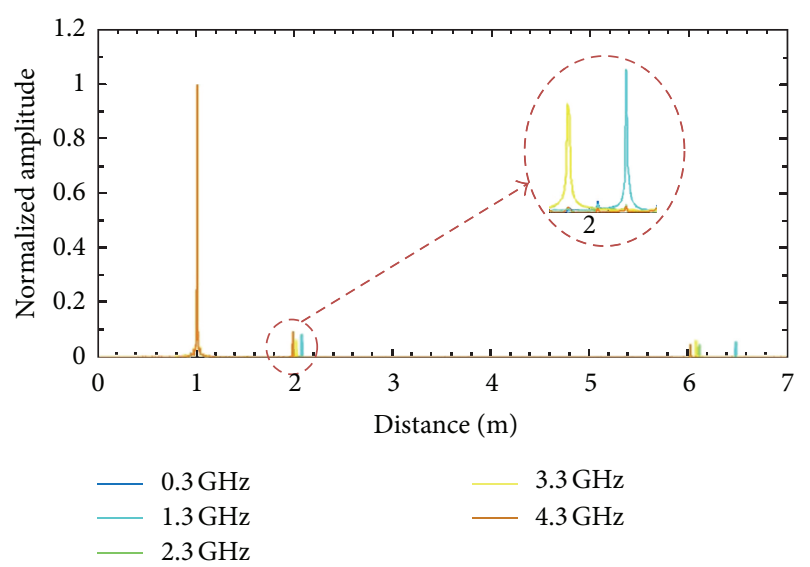

Figure 9: Shift effect on the retrieved signal peaks.

\section{Conclusion and Future Development}

The adoption of a Software Defined Radar system, used in conjunction with an orthogonal frequency-division multiplexing technique, has been discussed in this work. The advantageous features of Software Defined Radar technology, in terms of lowcost and easy implementation of signal processing algorithms, exploited together the adoption of multicarriers in the orthogonal frequency-division multiplexing method, to implement a multiband Software Defined Radar system able to accurately retrieve the geometrical and dielectric profiles of multilayer soils, with the further capability to reconstruct the frequency variation of permittivity. The proposed approach has been successfully validated on a two-layer soil structure. As a future work, a further extension of the method to a full dielectric characterization of media, including also the reconstruction of the imaginary part of the dielectric constant, will be developed. At this purpose, more accurate models for the multilayer structures and more efficient inversion algorithms for the dielectric characterization will be considered.

\section{Acknowledgment}

This work has been carried out under the framework of PON 01_01503 National Italian Project "Landslides Early Warning," financed by the Italian Ministry of Education, University and Research.

\section{References}

[1] G. Aloi, A. Borgia, S. Costanzo et al., "Software defined radar: synchronization issues and practical implementation," in Proceedings of the 4th International Conference on Cognitive Radio and Advanced Spectrum Management (CogART '11), Barcelona, Spain, October 2011.

[2] S. Costanzo, F. Spadafora, A. Borgia, O. H. Moreno, A. Costanzo, and G. Di Massa, "High resolution software defined radar system for target detection," Advances in Intelligent Systems and Computing, vol. 206, pp. 997-1005, 2013.
[3] S. Sen, M. Hurtado, and A. Nehorai, "Adaptive OFDM radar for detecting a moving target in urban scenarios," in Proceedings of the International Waveform Diversity and Design Conference (WDD '09), pp. 268-272, February 2009.

[4] S. Bausson and P. Forster, "Detection and pulse compression In passive radar with OFDM radio signals," in Proceedings of the 16th European Signal Processing Conference (EUSIPCO '08), Lausanne, Switzerland, 2008.

[5] M. Braun, C. Sturm, and F. K. Jondral, "Maximum likelihood speed and distance estimation for OFDM radar," in Proceedings of the IEEE International Radar Conference (RADAR '10), pp. 256-261, May 2010.

[6] C. Sturm, T. Zwick, and W. Wiesbeck, "An OFDM system concept for joint radar and communications operations," in Proceedings of the 69th IEEE Vehicular Technology Conference (VTC '09), April 2009.

[7] M. Fuhr, M. Braun, C. Sturmz, L. Reichardtz, and F. K. Jondral, "An SDR-based experimental setup for OFDM-based radar," in Proceedings of the Virginia Tech Symposium on Wireless Communications, Blacksburg, Va, USA, June 2012.

[8] S. Sen and A. Nehorai, "Adaptive design of OFDM radar signal with improved wideband ambiguity function," IEEE Transactions on Signal Processing, vol. 58, no. 2, pp. 928-933, 2010.

[9] N. Majurec, S. M. Sekelsky, S. J. Frasier, and S. A. Rutledge, “The advanced multi-frequency radar (AMFR) for remote sensing of clouds and precipitation," in Proceedings of the 11th Conference on Mesoscale Processes and the 32nd Conference on Radar Meteorology, pp. 1051-1057, Albuquerque, NM, USA, October 2005, paper no. P1R.6.

[10] P. V. Genderen, P. Hakkaart, J. V. Heijenoort, and G. P. Hermans, "A multi frequency radar for detecting landmines: design aspects and electrical performance," in Proceedings of the 31st European Microwave Conference, vol. 2, pp. 249-252, 2001.

[11] M. L. Bryan, "Interpretation of an urban scene using multichannel radar imagery," Remote Sensing of Environment, vol. 4, no. 1, pp. 49-66, 1975.

[12] B. R. Mahafza and A. Z. Elsherbeni, Simulations for Radar Systems Design, Chapman and Hall/CRC, 2003.

[13] C. Sturm, T. Zwick, and W. Wiesbeck, "Spectral estimationbased OFDM radar algorithms for IEEE 802.11a signals," in Proceedings of the IEEE Vehicular Technology Conference (VTC '12), 2012.

[14] N. R. Peplinski, F. T. Ulaby, and M. C. Dobson, "Dielectric properties of soils in the 0.3-1.3-GHz range," IEEE Transactions on Geoscience and Remote Sensing, vol. 33, no. 3, pp. 803-807, 1995.

[15] T. W. Miller, B. Borchers, J. M. H. Hendrickx, S. Hong, L. W. Dekker, and C. J. Ritsema, "Effects of soil physical properties on GPR for landmine detection," in Proceedings of the 5th International Symposium on Technology and the Mine Problem, 2002.

[16] M. C. Dobson, F. T. Ulaby, M. T. Hallikainen, and M. A. ElRayes, "Microwave dielectric behavior of wet soil-part II: dielectric mixing models," IEEE Transactions on Geoscience and Remote Sensing, vol. 23, no. 1, pp. 35-46, 1985.

[17] C. A. Balanis, Advanced Engineering Electromagnetics, John Wiley \& Sons, 1989. 

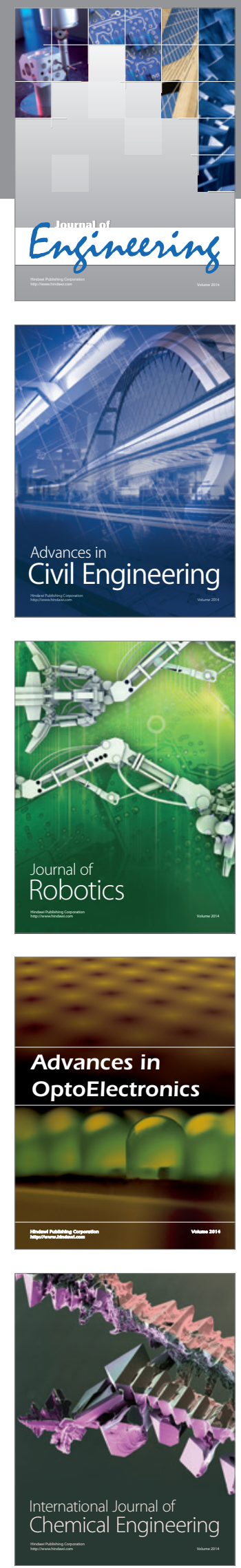

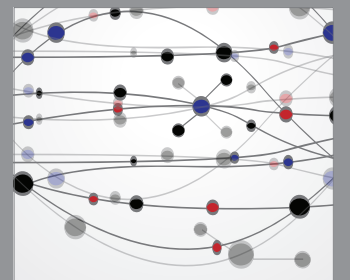

The Scientific World Journal
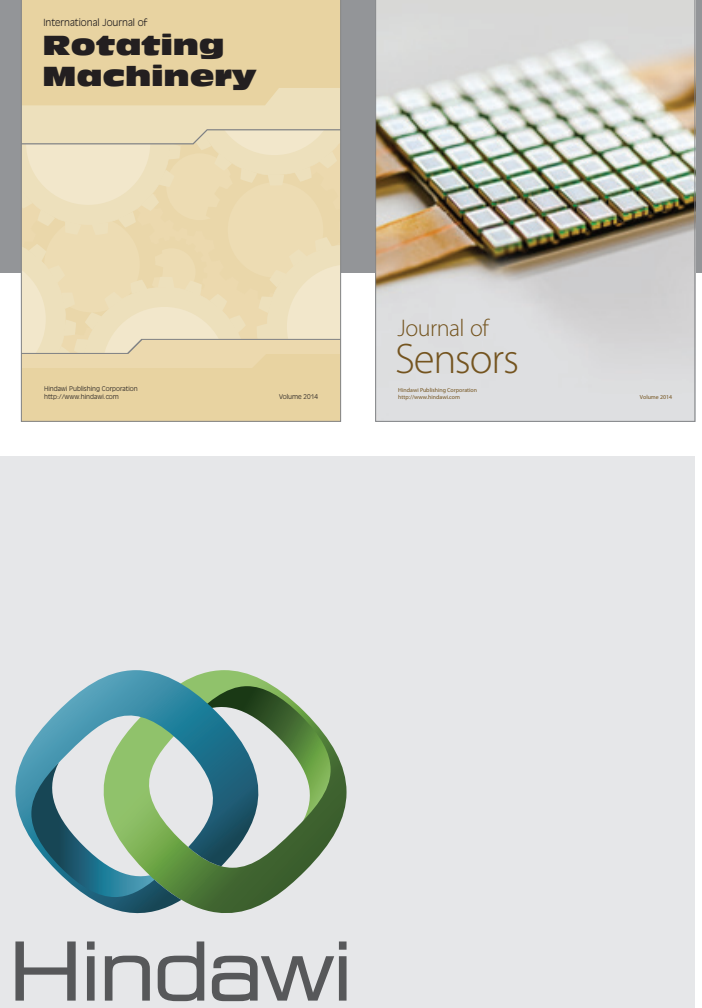

Submit your manuscripts at http://www.hindawi.com
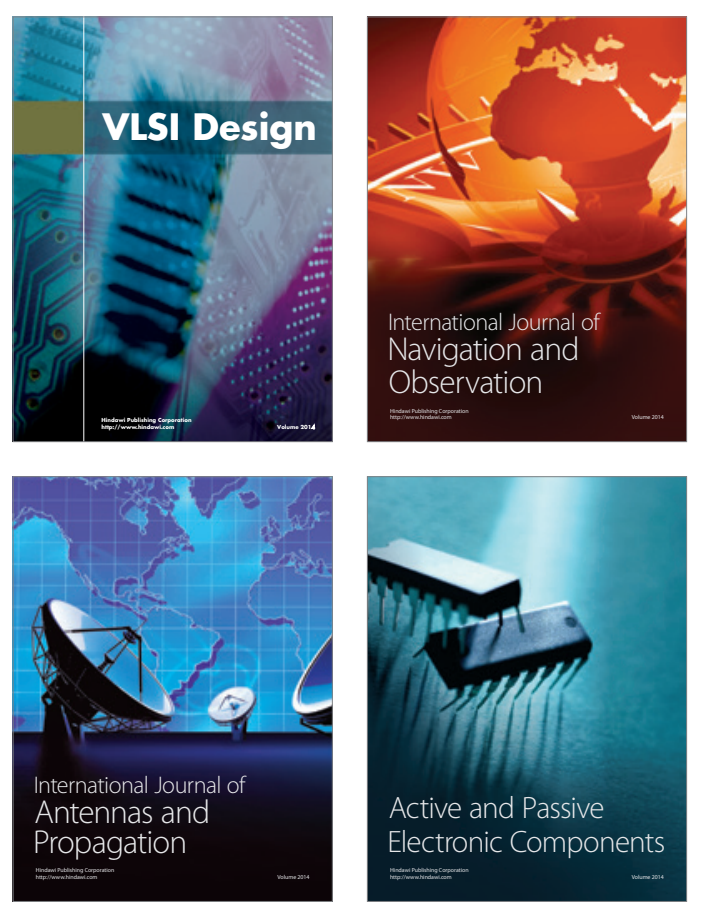
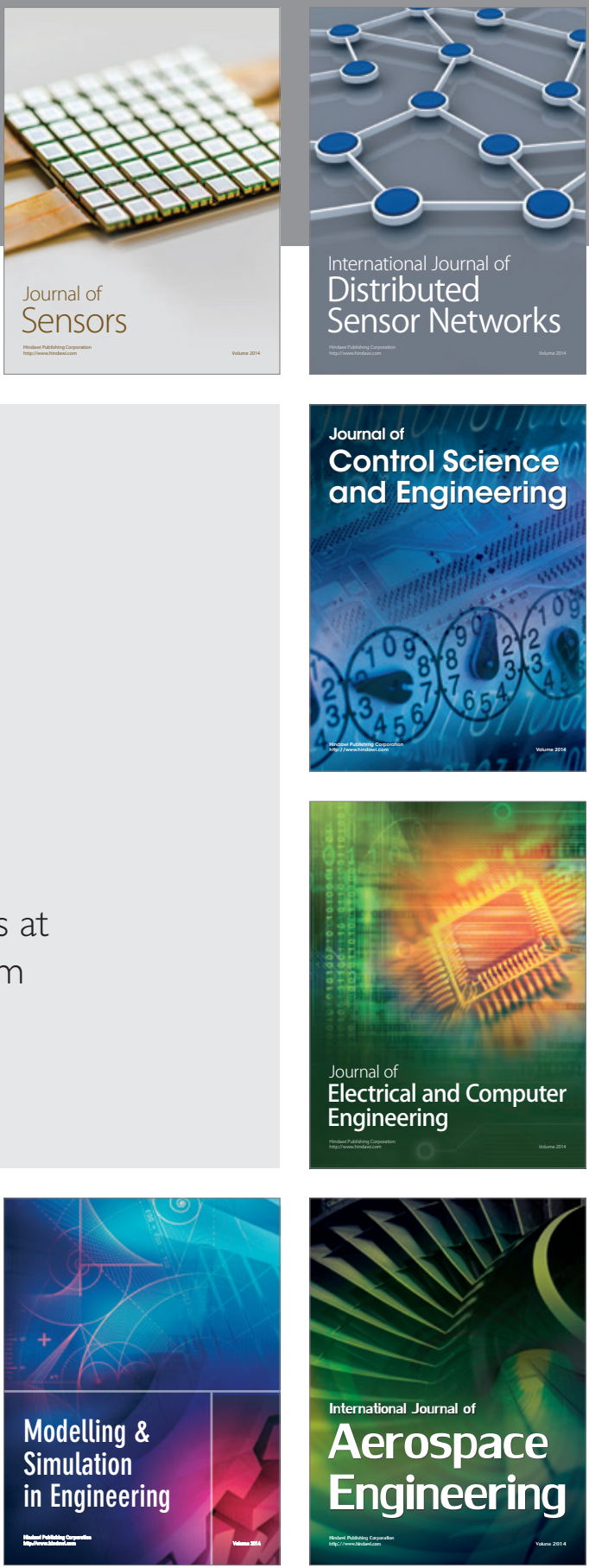

Journal of

Control Science

and Engineering
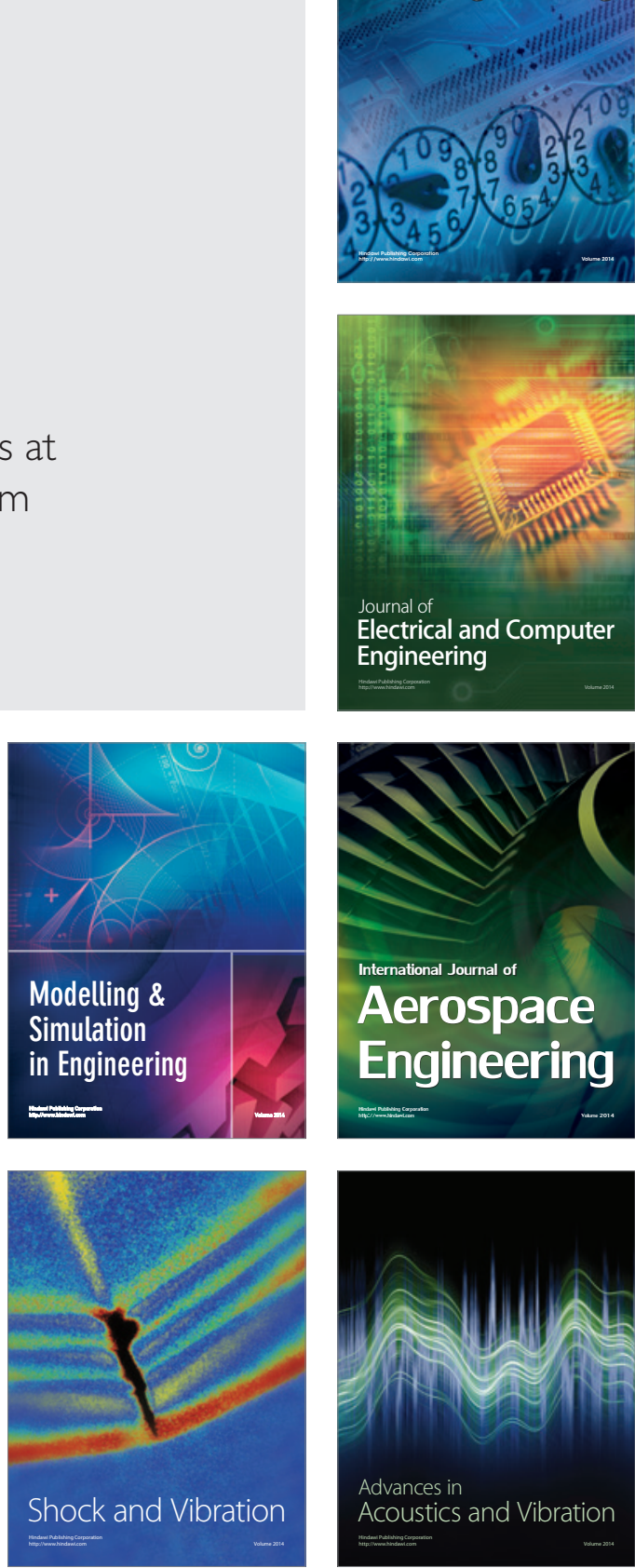